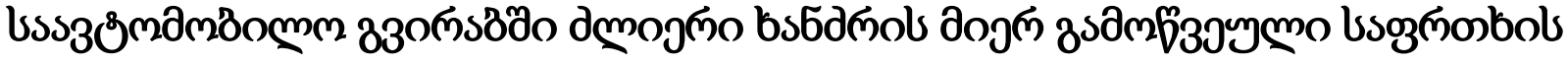 sढsmoso
}

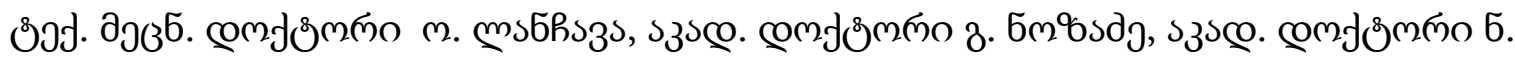

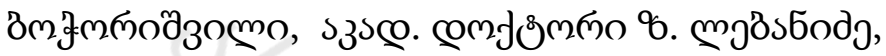

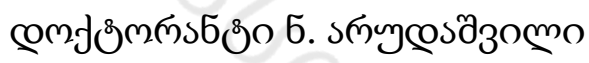

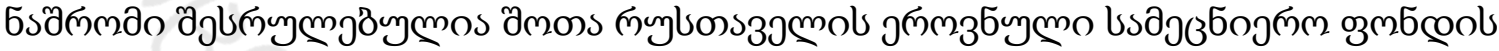

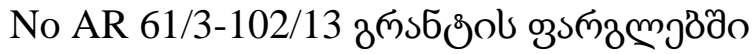

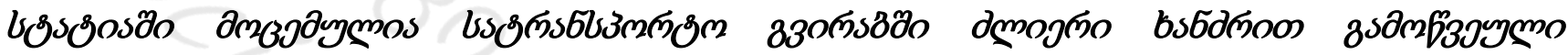

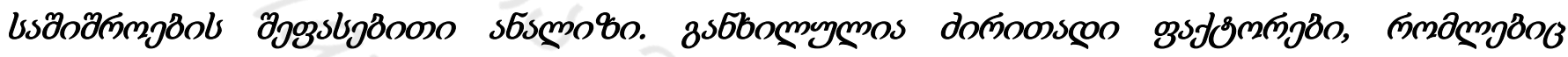

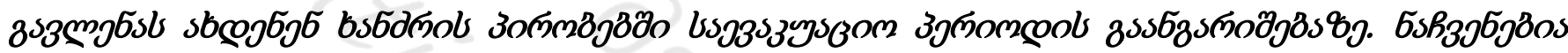

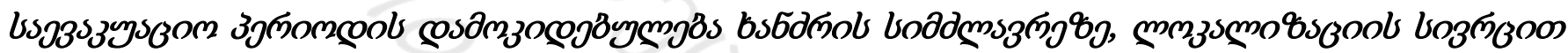
зుпs

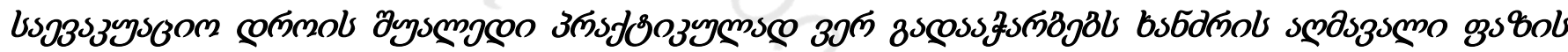

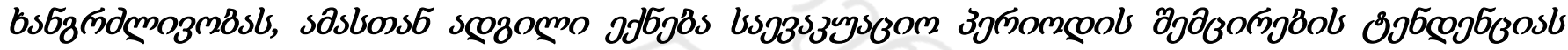

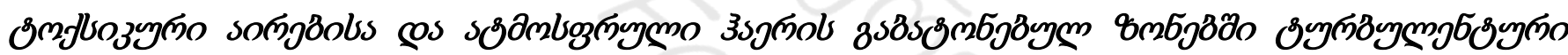

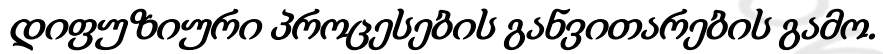

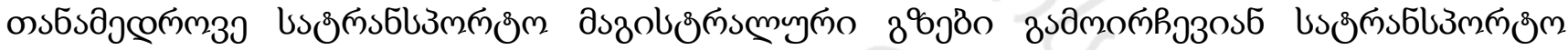

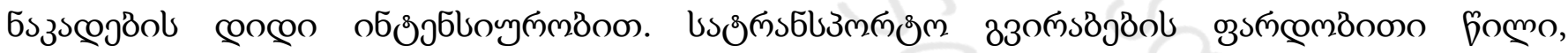

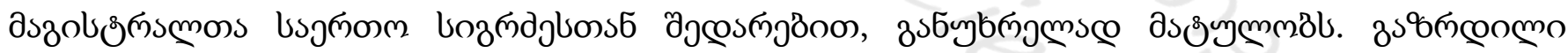

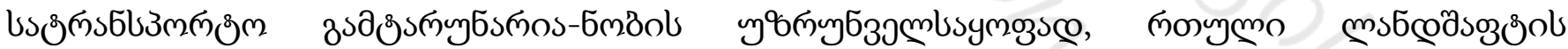

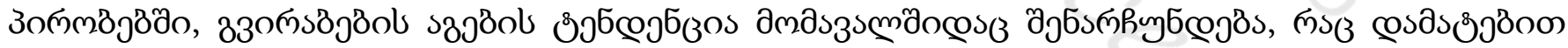

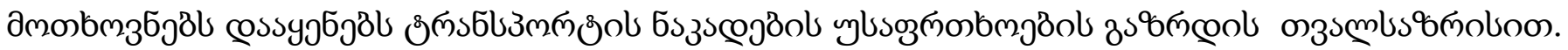

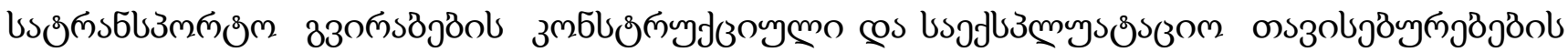

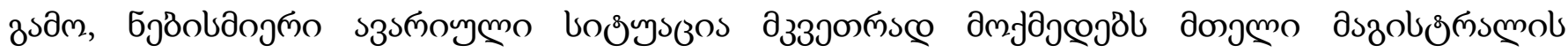

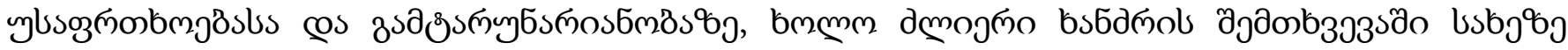

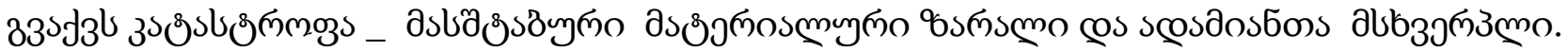




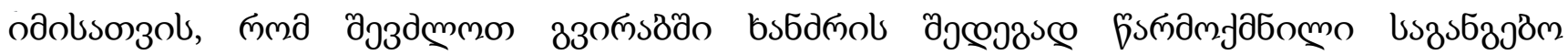

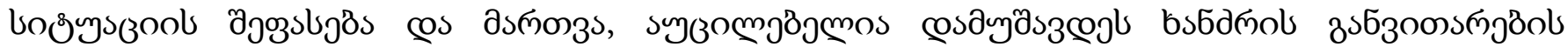

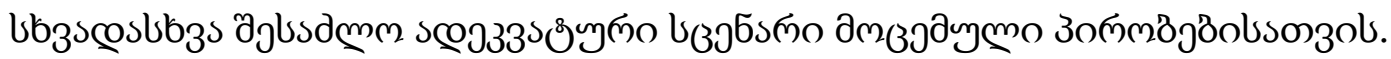

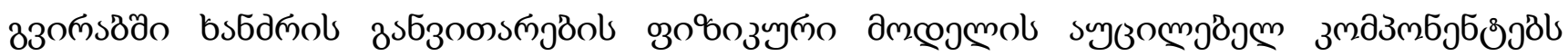

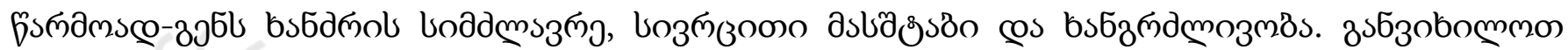

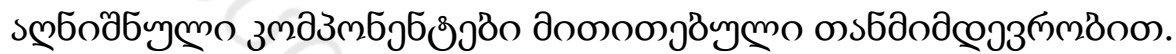

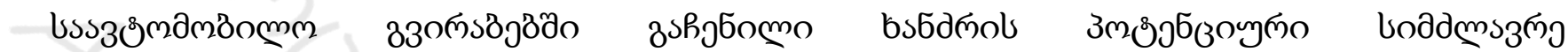

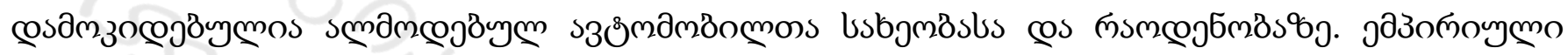

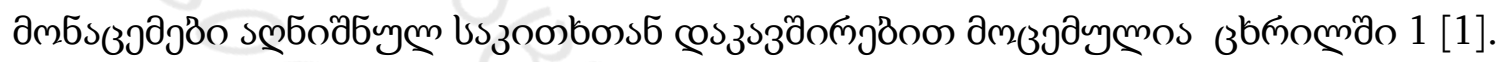

3bハомпо 1

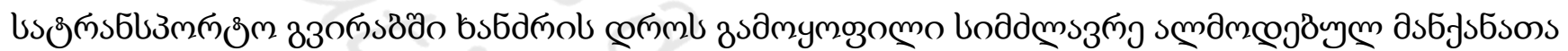
mozbzol aobjezom

\begin{tabular}{|c|c|}
\hline 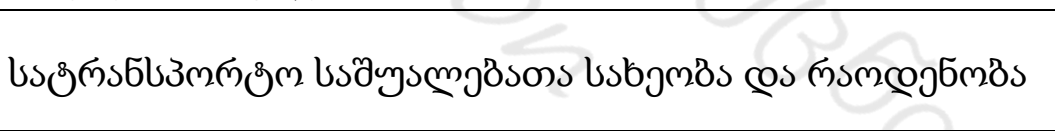 & 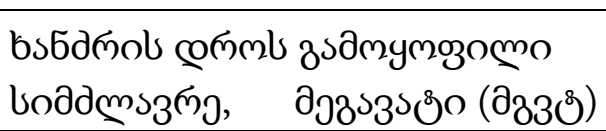 \\
\hline 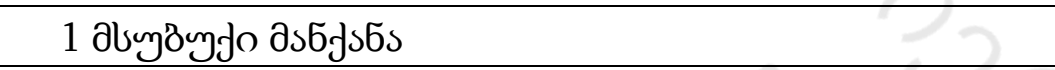 & 5 \\
\hline 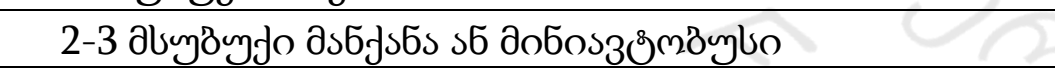 & $8 \_15$ \\
\hline 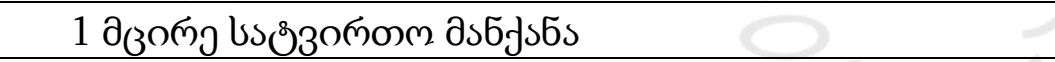 & 15_20 \\
\hline 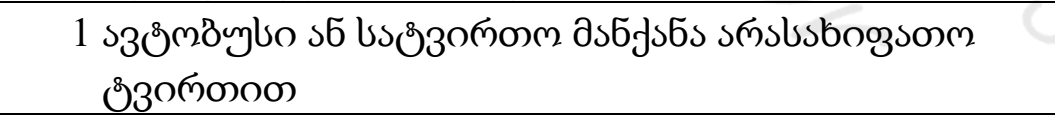 & 20_30 \\
\hline 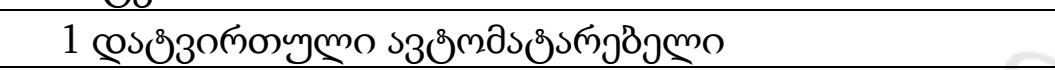 & 100 \\
\hline 1 8у6\%обд\%осо & 200_300 \\
\hline
\end{tabular}

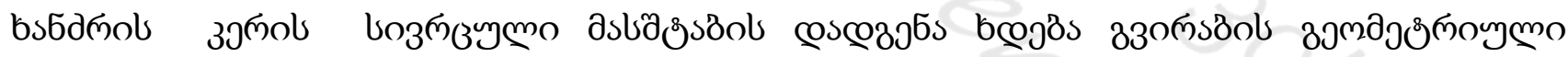

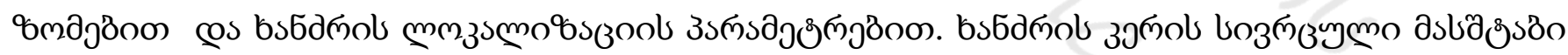

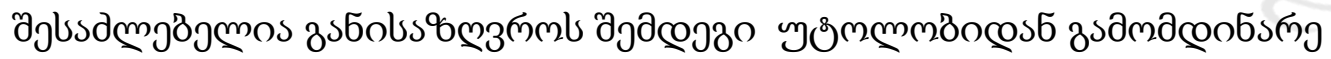

$$
V_{F} \leq V_{T}
$$

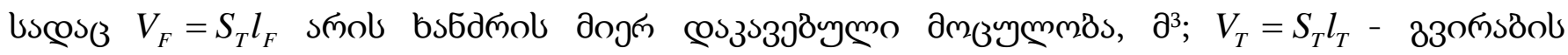

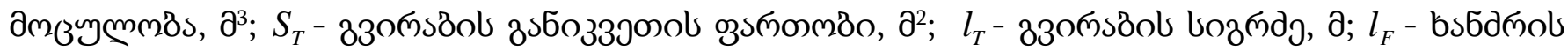

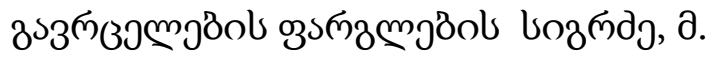




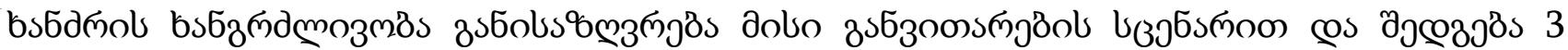

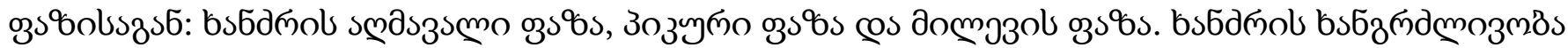

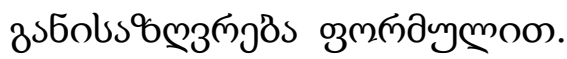

$$
T_{F}=t_{1 F}+t_{2 F}+t_{3 F},
$$

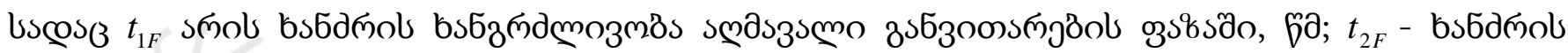

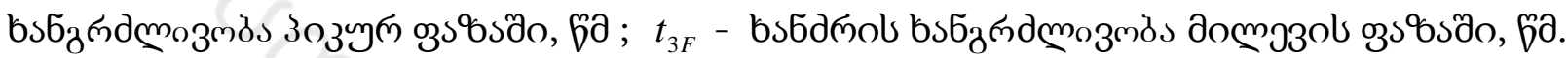

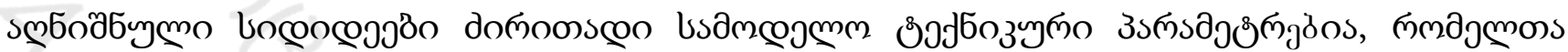

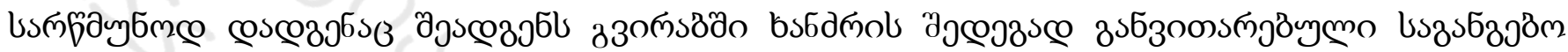

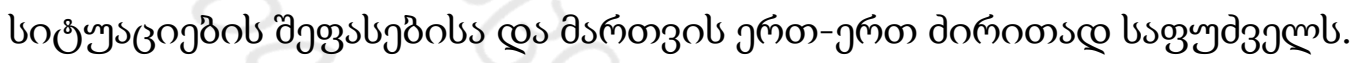

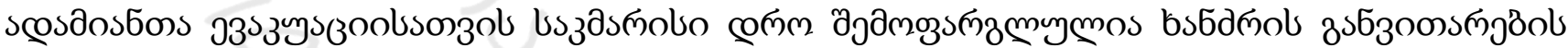

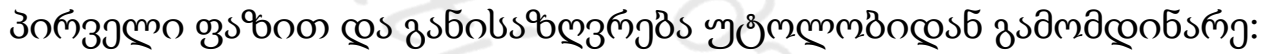

$$
t_{E V} \leq t_{1 F} .
$$

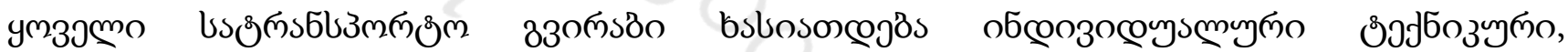

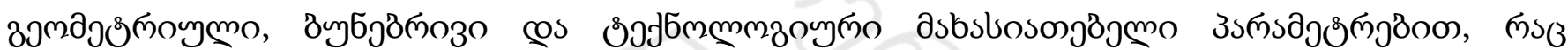

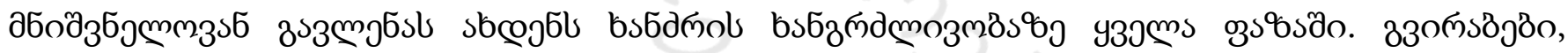

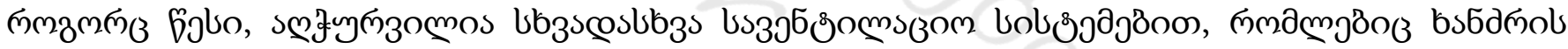

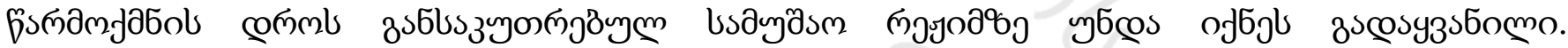

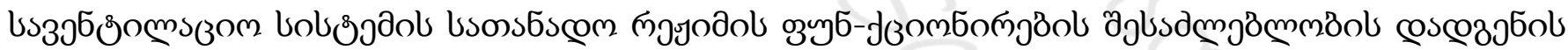

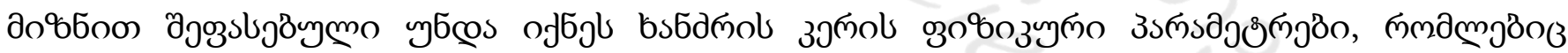

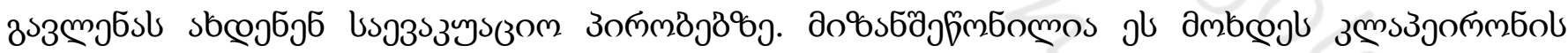

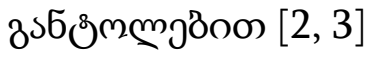

$$
P_{F} V_{F}=R T_{F},
$$

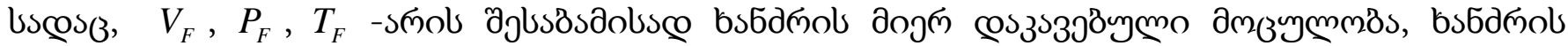

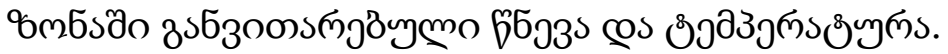

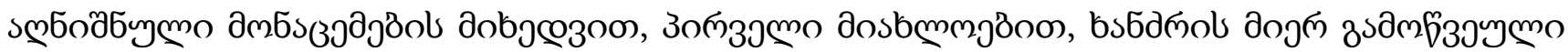

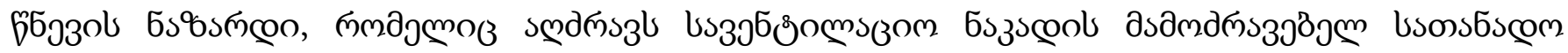




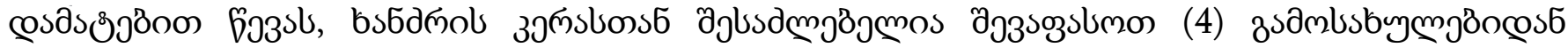

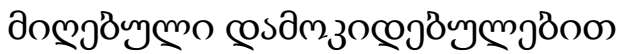

$$
\Delta P=\left(\rho-\rho_{0}\right) R \Delta T,
$$

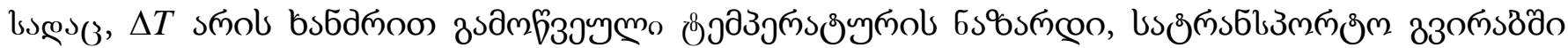

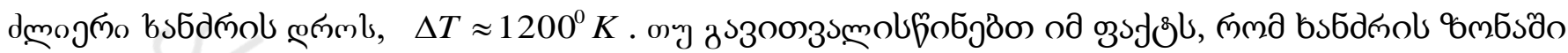

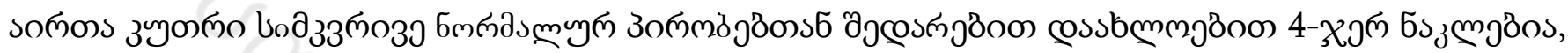

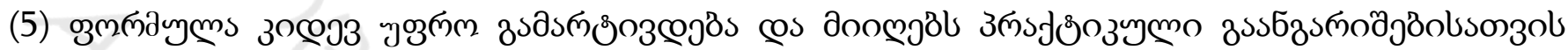

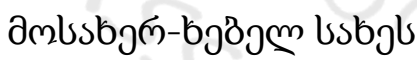

$$
\Delta P \approx 0,2-0,3 P_{0} \text {. }
$$

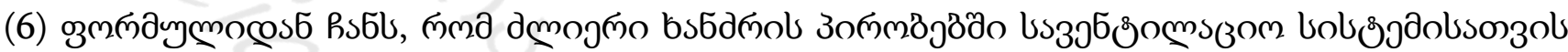

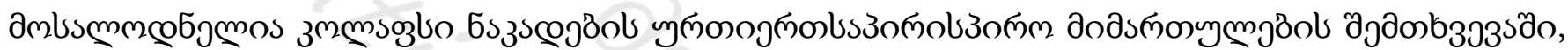

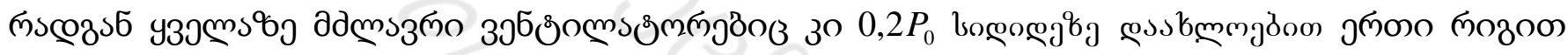

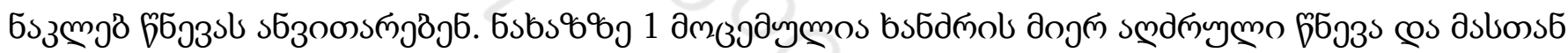

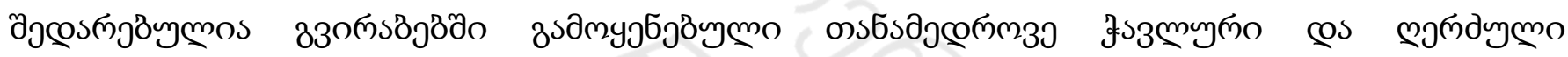

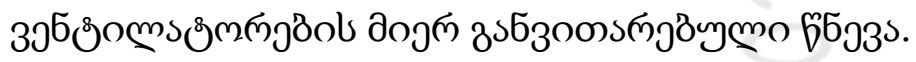

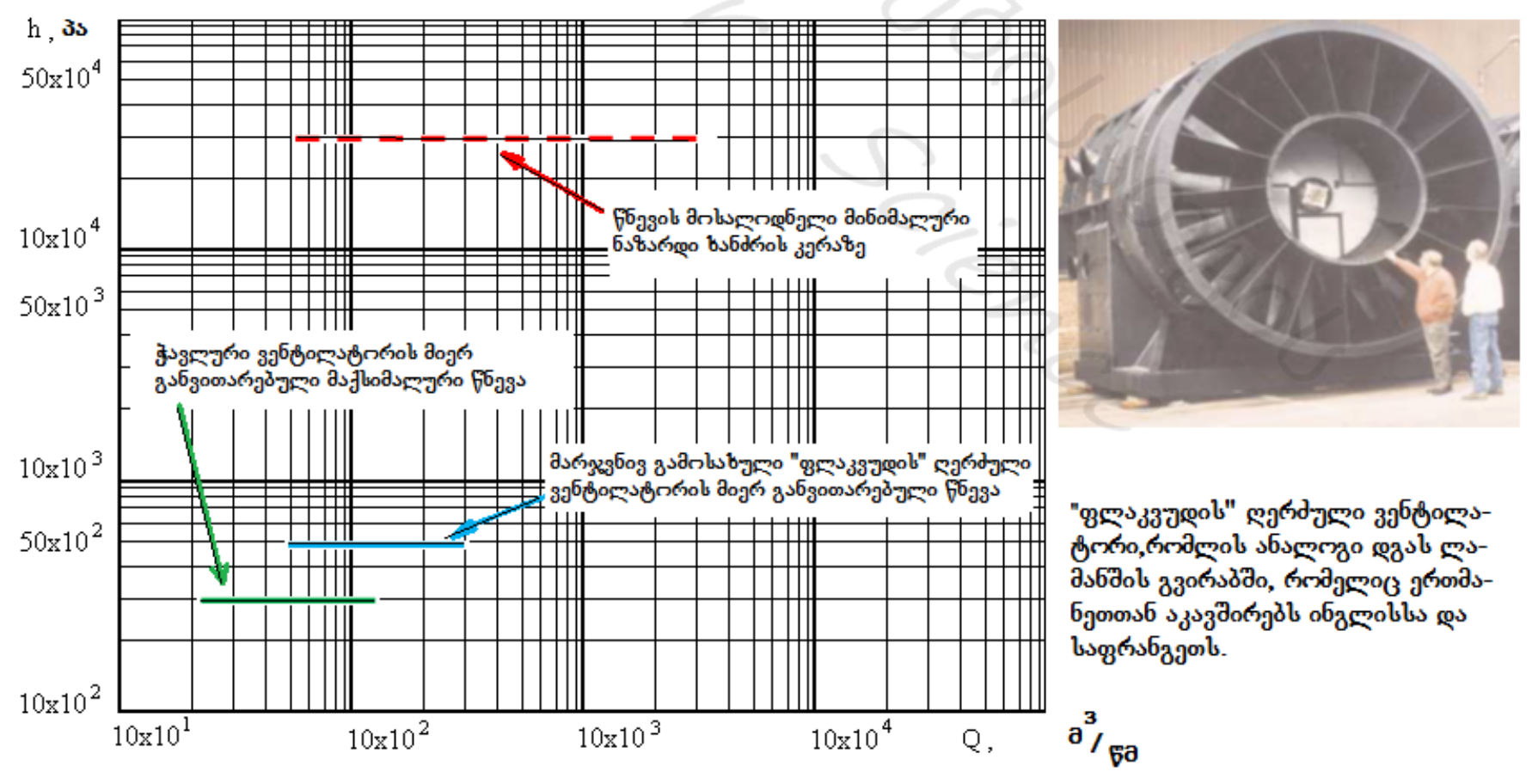




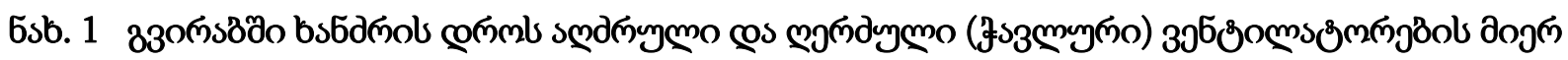

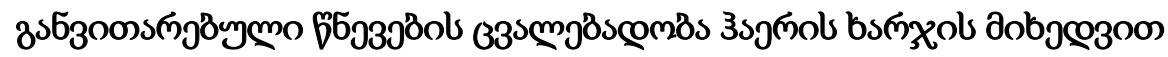

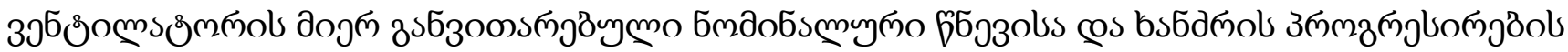

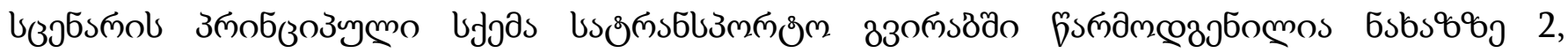

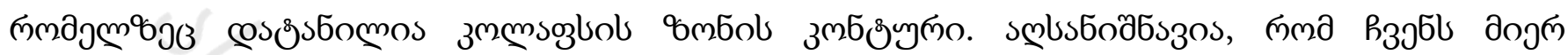

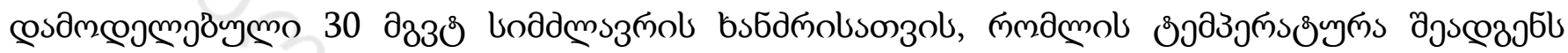

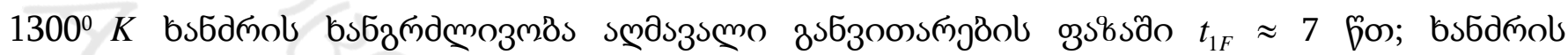

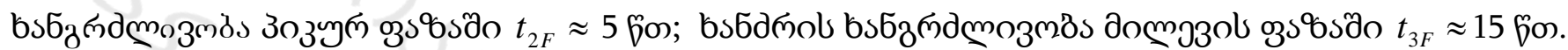

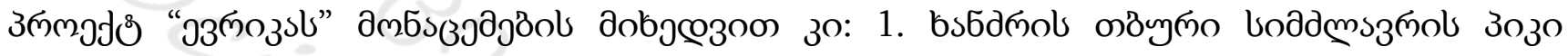

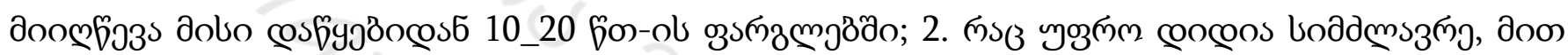

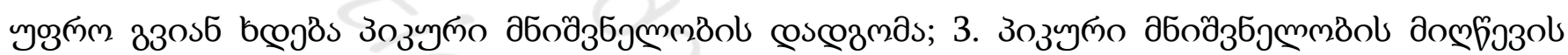

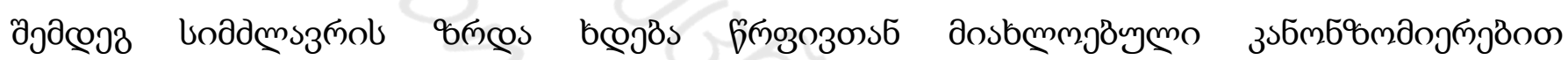

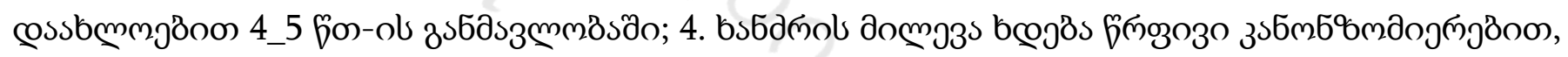

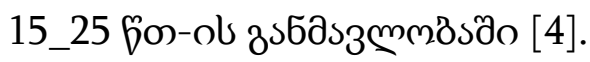

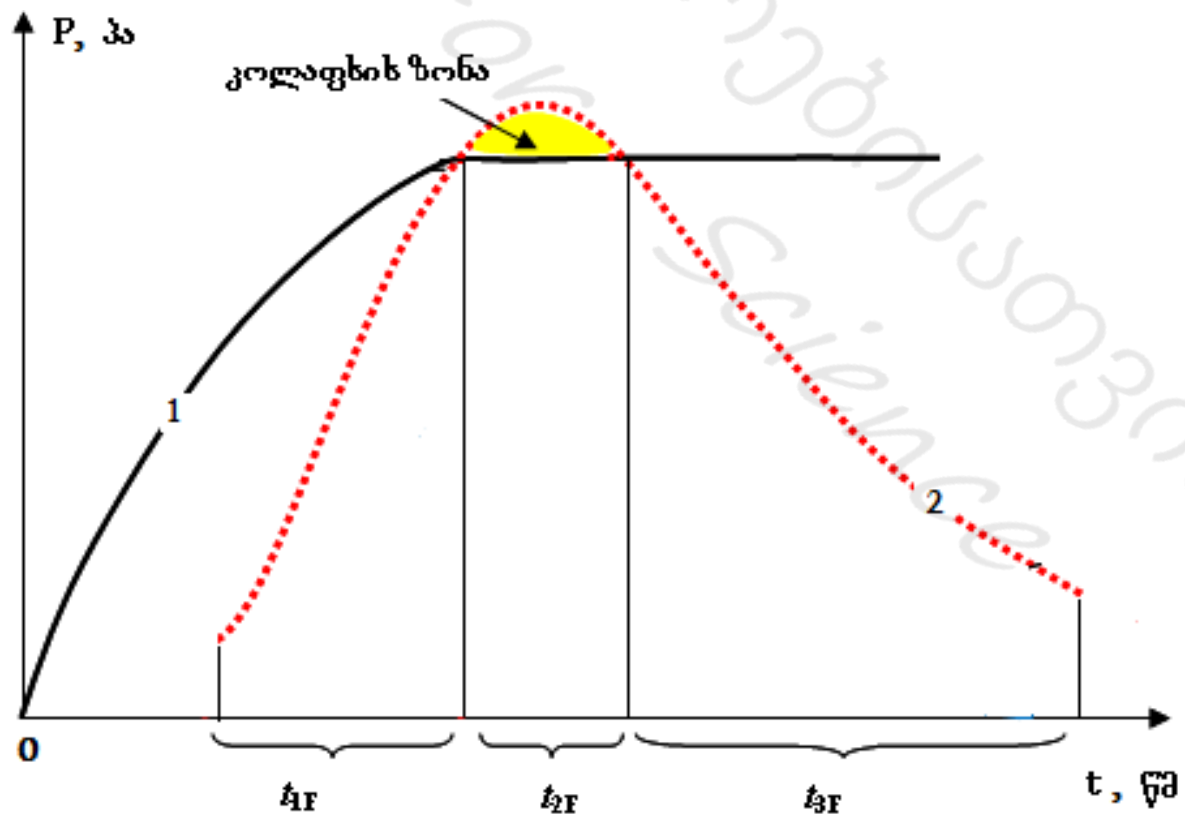




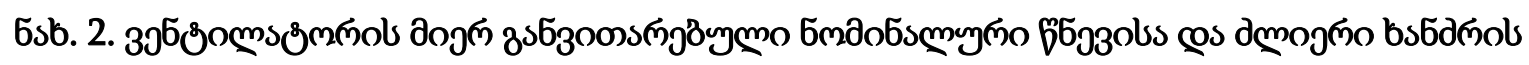

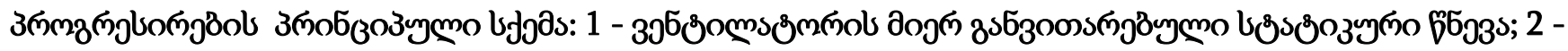

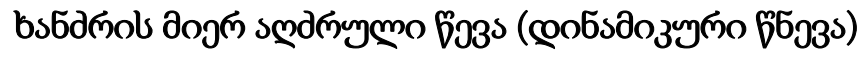

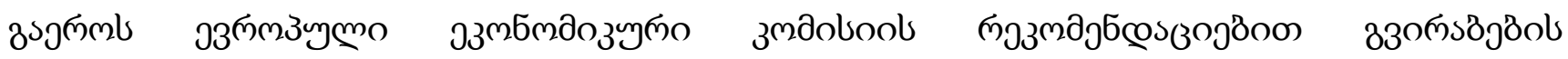

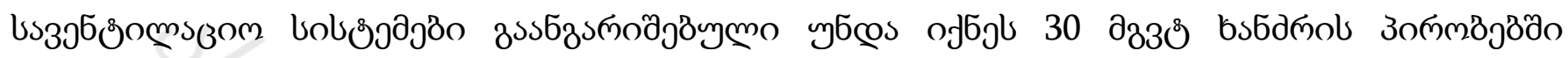

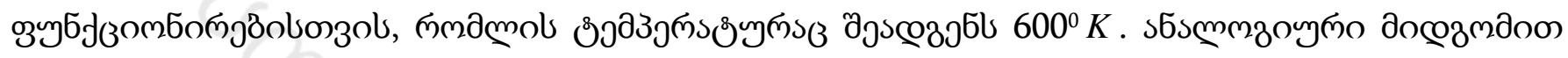

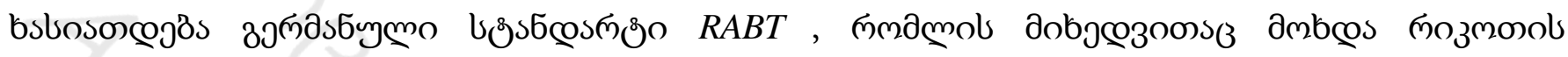

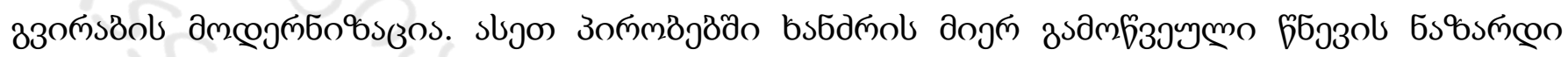

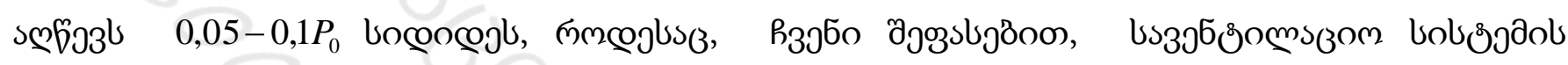
з замsoglo os

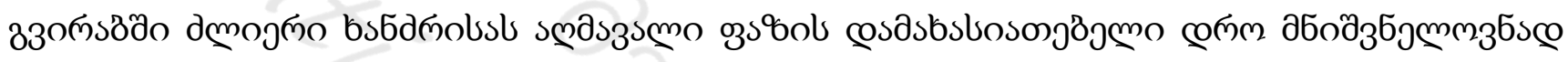

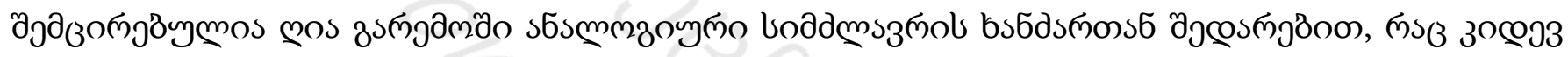

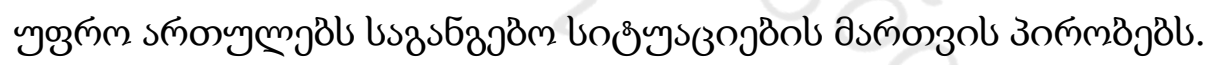

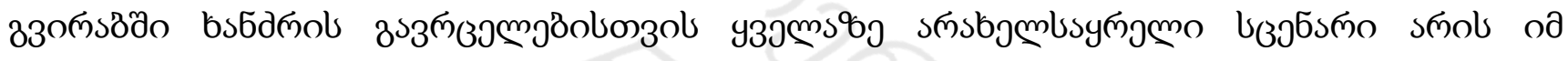

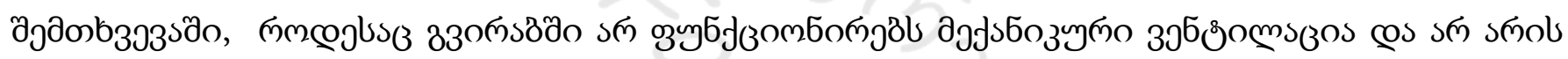

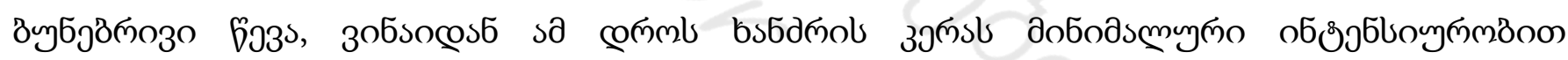

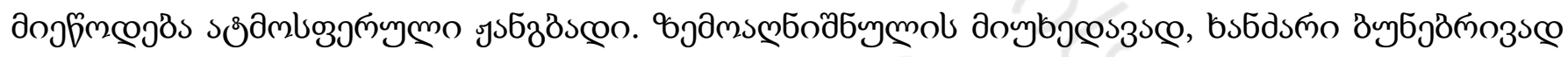

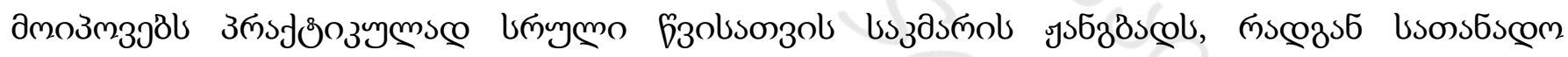

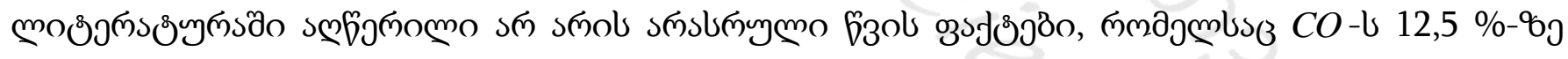

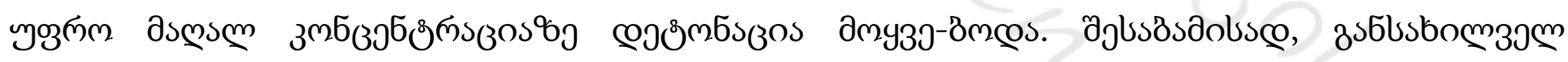

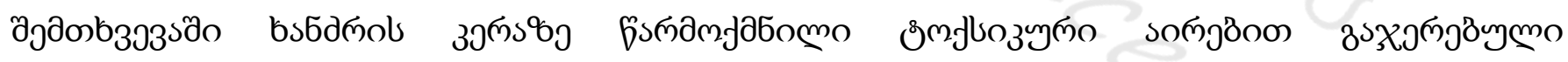

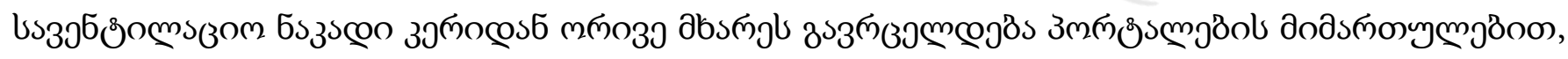

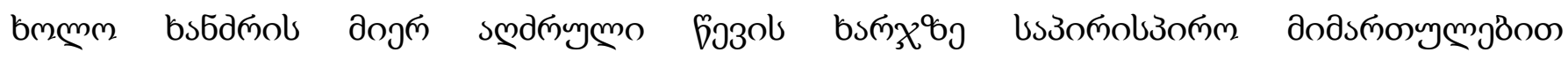

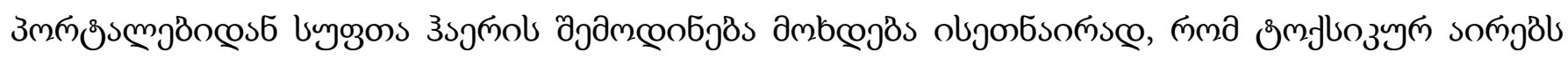

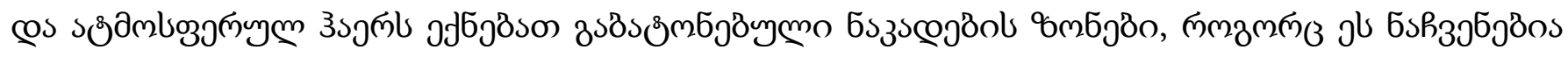
bstos $8 \%$ o 3. 


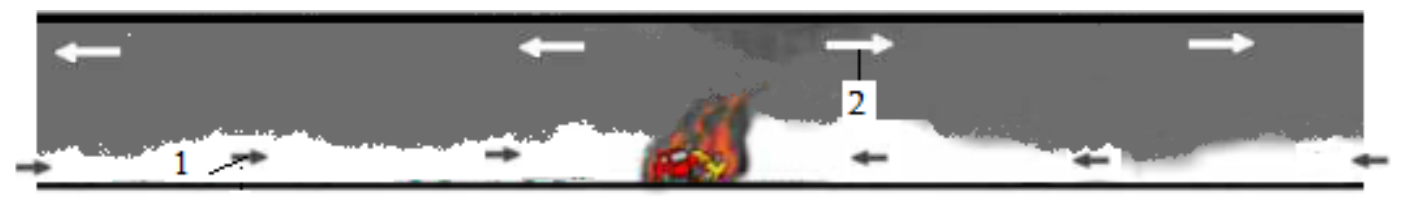

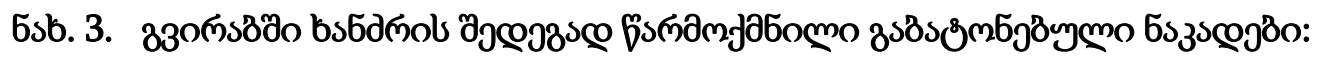

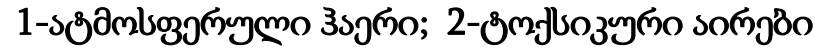

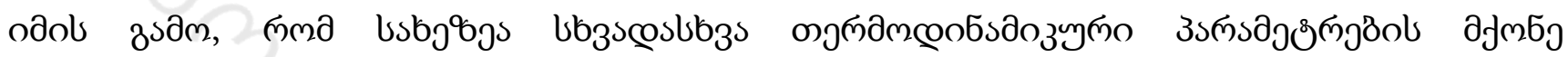

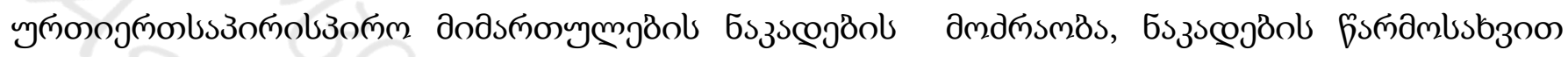

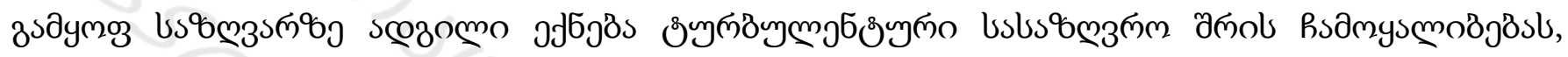

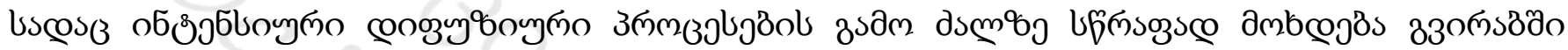

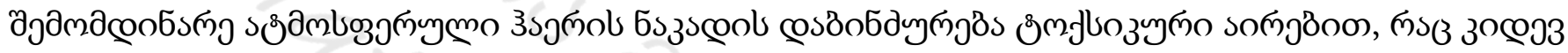

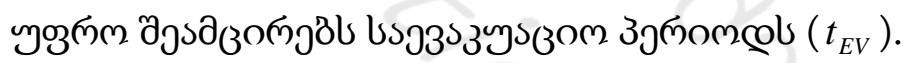

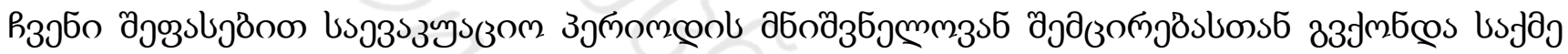

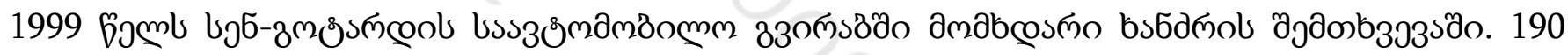

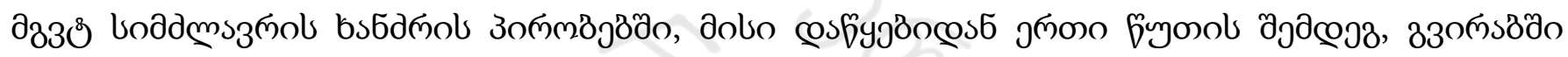

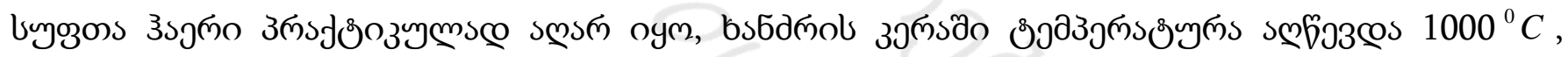

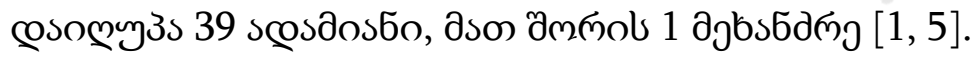

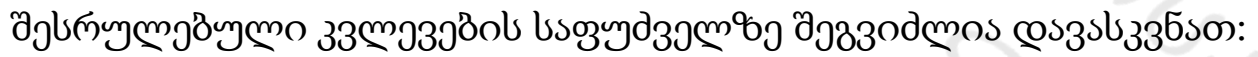

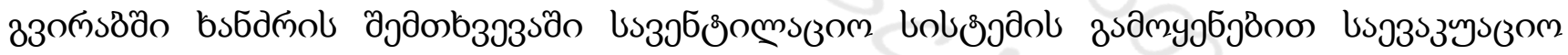

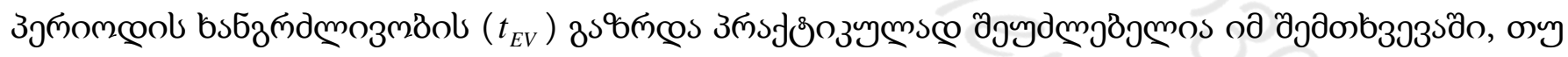

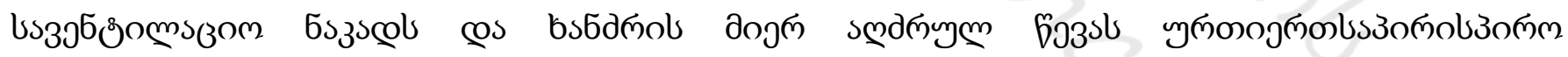

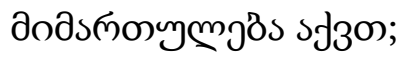

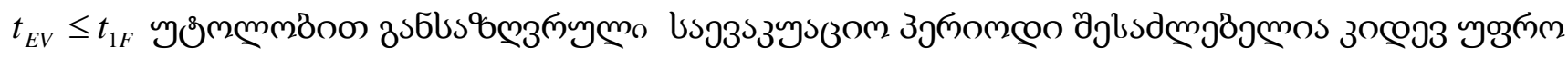

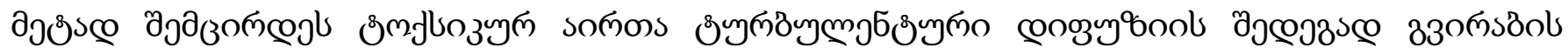

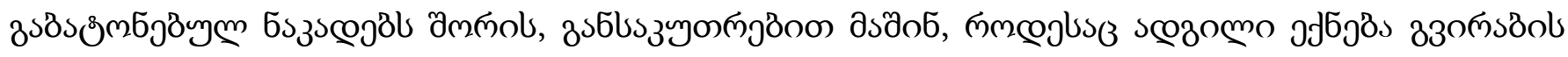

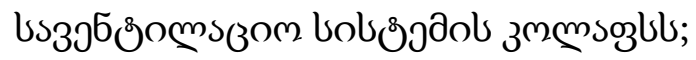




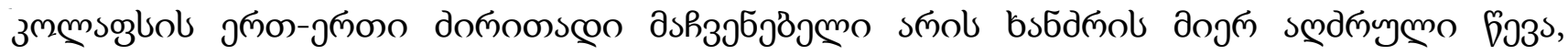

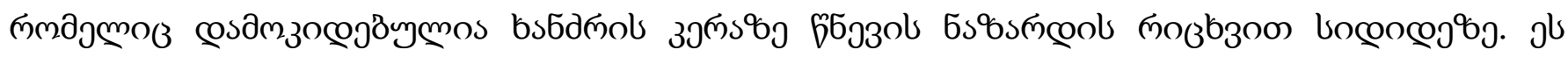

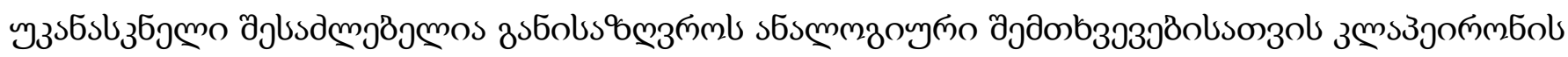

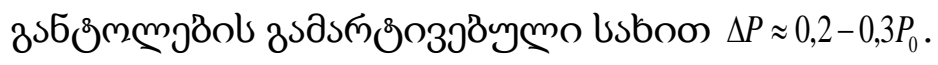

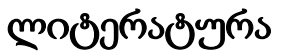

1. UN, Economic and Social Council, Economic Commission for Europe, Report TRANS/AC.7/9, 2001. p. 59.

2. O.Lanchava, N.Ilias, I. Andras, R.Moraru, I.Neag. On the Ventilation of Transport Tunnels in the Presence of a Strong (Heavy) Fire. Annals of the University of Petrosani, Petrosani (Romania), 2007, Vol. 9 (XXXVI), Part 1.pp. 219-227.

3. O.A. Lanchava. Hygroscopic heat and mass transfer in underground structures, GTU, Tbilisi, 1998, p. 272

4. A.Haack. Fire Protection in Traffic Tunnels: General Aspects and Results of the EUREKA Project, TUNNELING AND UNDERGROUND SPACE TECHNOLOGY, 1998, Volume 13, № 2. pp. 377-381.

5. UN, Economic and Social Council, Economic Commission for Europe, Report TRANS/AC.7/11, 2002. p. 6.

\section{HAZARD ANALYSIS DUE TO THE INFLUENCE OF THE STRONG FIRES IN THE ROAD TUNNELS}

\section{LANCHAVA O., NOZADZE G., BOCHORISHVILI N., LEBANIDZE Z., ARUDASHVILI N.}

In this article presented an analysis of the hazards caused by the influence of strong fires in the road tunnels. Have been considered the main factors that affect the reliability of the results of definition of the period of time during which there is the possibility of evacuation of people. The dependence of the period of time marked by the characteristic parameters of fire: from the power of spatial localization and duration of the ascending phase of its development. The paper noted that the duration of the evacuation period, will be less than the duration of the ascending phase of development of a fire and also noted that this period tends to narrowing due to the influence of turbulent diffusion fluxes. In this paper it is assumed that the turbulent diffusion fluxes occur between the zones in which the ruling are toxic carbon gases of fire and fresh ventilation air flow. 